\title{
COLCHICINE-INDUCED ABNORMAL MEIOTIC CHROMOSOMAL SEGREGATION IN PRIMARY OOCYTES OF THE CHINESE HAMSTER
}

\author{
Part I. NONDISJUNCTION
}

\author{
Shigeki Sugawara and Kazuya Mikamo \\ Department of Biological Sciences, Asahikawa Medical College, \\ 4-5 Nishikagura, Asahikawa 078-11, Japan
}

\begin{abstract}
Summary In vivo genome mutagenicity of colchicine in the primary oocytes of the Chinese hamster was analyzed at a selected dose that did not completely arrest the formation of the first meiotic spindle, but exhibited a remarkable induction of nondisjunction, very possibly as the result of the inhibition of tubulin polymerization.

A single dose of $3 \mu \mathrm{g} / \mathrm{g}$ b.w. colchicine was intraperitoneally administered to females with a normal estrous cycle at the onset of the formation of the first meiotic spindle. Chromosome analysis of a total of 2,124 secondary oocytes revealed that the incidence of aneuploids increased significantly $(\mathrm{p}<0.001)$ in the experimental group $(25.9 \%, 99$ out of 382$)$ as compared with the control group $(2.0 \%, 35$ out of 1,742$)$. The minimum genome mutagenicity of colchicine caused nondisjunction in one or two bivalents and eventually induced a large number of aneuploid secondary oocytes within haploid range.

The condition of the spindle in oocytes aged by pre- and postovulatory overripeness, both of which have been known to induce meiotic chromosomal nondisjunction, were discussed in relation to tubulin polymerization.
\end{abstract}

\section{INTRODUCTION}

It has been confirmed that colcemid induces aneuploidy in mammalian somatic cells in vitro, owing to its deleterious effect on the mitotic spindle (Cox and Puck, 1969; Kato and Yoshida, 1970, 1971; Cox, 1973). Prior to this, in vivo administration of colchicine has been found to suppress the second meiotic chromosomal segregation of oocytes, thereby inducing triploids in rodents (Edwards, 1954, 1958; Piko and Bomsel-Helmreich, 1960; McGaughey and Chang, 1969). These studies suggested that aneuploids were also produced, but unfortunately, the chromosomal

Received April 21, 1980 
technique was not sufficiently developed yet to demonstrate with accuracy the genome mutagenicity of colchicine. Today, however, it is possible to undertake a much more informative experiment, since the chromosomal technique for mammalian oocytes has recently been greatly advanced (Kamiguchi et al., 1976, 1978).

Considering the recent achievements in the heteromorphic marker chromosome studies which have shown that nondisjunction seems to occur more frequently in meiosis I than in meiosis II of oocytes (Jacobs and Morton, 1977; Niikawa et al., 1977; Mattei et al., 1979), we attempted to examine the effect of colchicine on meiosis I of oocytes in vivo. The present paper describes the genome mutagenic effect of colchicine at a selected dose that did not completely arrest the formation of the first meiotic spindle of the Chinese hamster's oocytes, but exhibited a remarkable induction of nondisjunction.

\section{MATERIALS AND METHODS}

Virgin female Chinese hamsters aged 5-8 months were used. Under a constant laboratory condition, $14 \mathrm{hr}$ illumination from 5:00 to 19:00, temperature at $23 \pm 2{ }^{\circ} \mathrm{C}$ and humidity between $50-60 \%$, they maintained a stable 4-day estrous cycle. The surge of LH occurred at 14:00-15:00 of the day of proestrum and ovulation did at 3:30-4:30 the following morning. A single dose of $3 \mu \mathrm{g} / \mathrm{g} \mathrm{b} . \mathrm{w}$. colchicine was intraperitoneally injected to the females at 17:30 of the day of proestrum. We could be certain that the eggs were exposed to the chemical at the onset of spindle formation and thereafter. At about 9:00 of the next day, i.e., of the day of estrum, secondary oocytes were collected from the ampullar region of oviducts.

Chromosomal preparations were made by the method reported previously by us (Kamiguchi et al., 1976, 1978). The technique has been elaborated so as to avoid rupturing the plasma membrane of oocytes, in order to minimize the rate of accidental loss of chromosomes. Using this method, chromosomes of oocytes can be properly spread within flattened dry ooplasm without being scattered. Thus, more than $90 \%$ of the secondary oocytes were karyotyped successfully. In the Chinese hamster, metaphase II consists of eleven dyads which can be divided into four morphologically distinguishable groups, i.e., two large metacentric (Group A), three medium metacentric (Group B), three medium acrocentric (Group C) and three small metacentric chromosomes (Group D). Aberrant dyads were identified according to the karyologic system.

\section{RESULTS}

The incidence of aneuploid oocytes in the experimental group was much higher $(\mathrm{p}<0.001)$ than in the control group (Table 1). Thus, the induction of nondisjunction by colchicine in the first meiotic division of oocytes was unequivocally

Jpn. J. Human Genet. 
Table 1. Incidence of aneuploid oocytes II of the Chinese hamsters injected with $3 \mu \mathrm{g} / \mathrm{g}$ b.w. colchicine.

\begin{tabular}{lccccc} 
& $\begin{array}{c}\text { No. of } \\
\text { animals }\end{array}$ & $\begin{array}{c}\text { No. of oocytes } \\
\text { collected } \\
(\text { mean) }\end{array}$ & $\begin{array}{c}\text { Total no. of } \\
\text { eggs karyotyped }\end{array}$ & $\begin{array}{c}\text { Normal } \\
(\%)\end{array}$ & $\begin{array}{c}\text { Aneuploid } \\
(\%)\end{array}$ \\
\hline Control group & 254 & $\begin{array}{c}1,912 \\
(7.5)\end{array}$ & $\begin{array}{c}1,742 \\
(91.1)^{*}\end{array}$ & $\begin{array}{c}1,707 \\
(98.0)\end{array}$ & $\begin{array}{c}35 \\
(2.0)\end{array}$ \\
Experimental group & 55 & $\begin{array}{c}316 \\
(7.6)\end{array}$ & $(91.8)^{*}$ & $(74.1)$ & $(25.9)$ \\
\hline$\chi^{2}$-test & & & & $p<0.001$ \\
\hline
\end{tabular}

* Rate of successful chromosome analysis.

** Giant diploid oocytes are excluded ( 5 cases from control group, 1 case from experimental group), but the diploid oocytes of normal size which are caused by the suppress of first polar body emission are included ( 2 cases in the experimental group).

proven. It was also found that the nondisjunction took place in every group of the karyologic system, A, B, C, and D (Fig. 1).

When these aneuploids were classified according to their chromosome numbers, it was evident that their frequency distributions differed remarkably between the experimental and control groups, as shown in Fig. 2. In the control group, the majority of the aneuploids had only one aberrant dyad, either superfluous or missing, and the incidence of hyperhaploids was not significantly different from that of hypohaploids, although the latter anomalies showed a somewhat higher incidence.

In the experimental group, as far as the aneuploids within haploid range having one or two aberrant dyads were concerned, there was a relation similar to that in the control group between the incidences of hyper- and hypohaploids. Evidently, however, both types of abnormal oocytes were much more frequent than in the control group. On the other hand, as to aneuploids with many aberrant dyads, the incidence of hypohaploids predominated significantly over that of hyperhaploids. Especially, the former anomalies with a very small number of dyads were seen frequently. It was found that their occurrence seemed to be related to the formation of a giant first polar body/bodies which were especially frequent in the experimental group. The mechanism by which a large number of dyads were lost from rather than added to oocytes will be demonstrated and discussed in Part II of the paper, in relation to the formation of the giant polar body/bodies.

\section{DISCUSSION}

Colchicine has recently been proven to inhibit the polymerization of tubulin, thereby to block the formation of the spindle microtubules in sea urchins (Wang et al., 1977). Our experiment strongly suggested that colchicine arrested the formation of first meiotic spindle microtubules when administered in vivo to the primary 


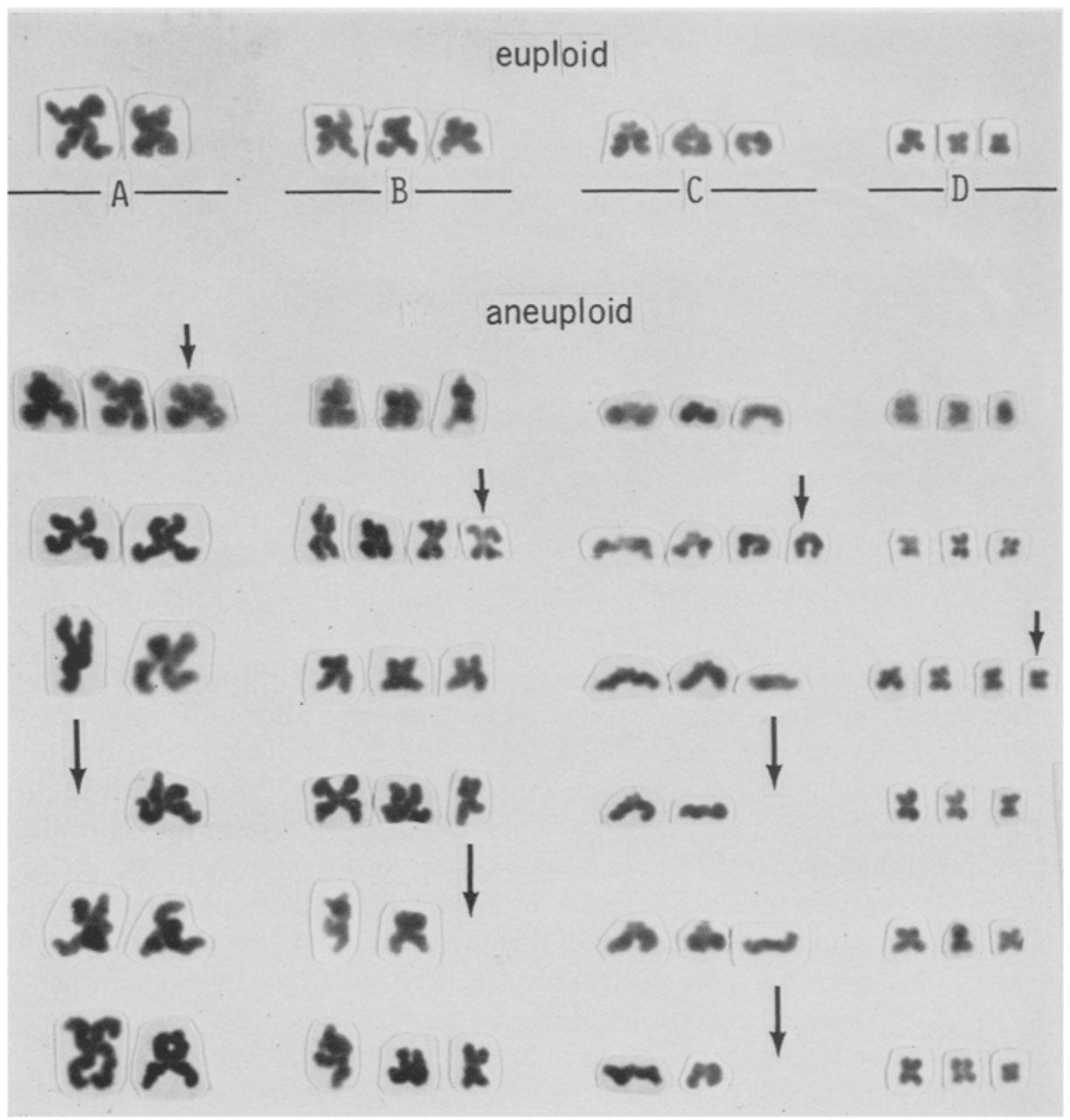

Fig. 1. The karyotype of a normal secondary oocyte of the Chinese hamster and those of aneuploid oocytes induced by a single dose of $3 \mu \mathrm{g} / \mathrm{g}$ b.w. colchicine which was injected intraperitoneally at the onset of formation of the first meiotic spindle. Note that the nondisjunction took place in every group, A, B, C, and D. Arrows indicate either missing or extra chromosomes.

oocytes immediately after germinal vesicle breakdown, that is, at about the time when the microtubule formation had begun.

It was shown that the minimum genome mutagenicity of colchicine caused nondisjunction in one or two bivalents and eventually induced a large number of aneuploid oocytes within haploid range. It is conceivable that the inhibition of the formation of a small number of microtubules may bring about the above results.

It has been demonstrated that meiotic nondisjunction could be associated with defect of spindle fibers (microtubules) in the first and second divisions of the eggs 


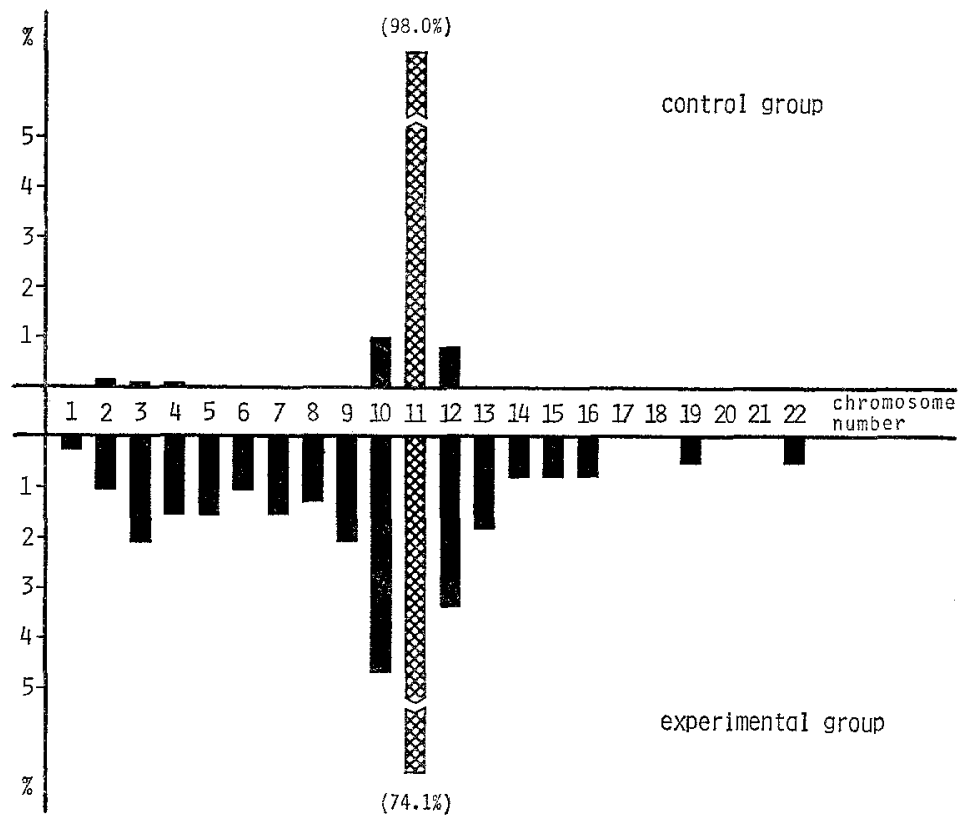

Fig. 2. The frequency distributions of aneuploid secondary oocytes of the control and the colchicine-treated group. Note the significantly higher incidence of aneuploids in the latter group and that in the same group hypohaploids are much more numerous than hyperhaploids.

of Xenopus laevis which had been aged by preovulatory overripeness (Mikamo, $1968 \mathrm{a}, \mathrm{b})$. Later, the same aging of primary oocytes was proven to cause aneuploidy in rats (Mikamo and Hamaguchi, 1975; Kamiguchi et al., 1979). The defect of microtubules could have been interpreted as the inhibition of tubulin polymerization which was caused by the unusually prolonged retention of the eggs at germinal vesicle stage within mature follicles. On the other hand, the nondisjunction caused by postovulatory overripeness occurring during the second meiotic division (Witschi and Laguens, 1963) might be resulted from depolymerization of tubulin in the once normally established spindle of secondary oocytes. Therefore, the abnormal condition of microtubules in the experiments with secondary oocytes that were cited before (Edwards, 1954, 1958; Piko and Bomsel-Helmreich, 1960; McGaughey and Chang, 1969) is much alike that following postovulatory aging, while that in the present experiment with primary oocytes is comparable with the situation occurring in the preovulatory aging oocytes. It is surmised that meiotic nondisjunction can be induced by chemical compounds similar to colchicine through their effect on tubulin polymerization. Many of the compounds with a trimethoxybenzene ring are known to have such an effect (Harrisson et al., 1976; Wang et al., 1977). 
Acknowledgements We are thankful to Dr. Y. Kamiguchi, Messrs. K. Funaki and N. Koide for their collaboration during the investigation. We are much obliged to Misses Y. Watanabe and Y. Baba who took care of our colony of Chinese hamsters.

Supported in part by grants from the Ministry of Education for Scientific Research and also for Developmental Scientific Research, and by grants from the Ministry of Health and Welfare for Handicapped Children, Japan.

\section{REFERENCES}

Cox, D.M. 1973. A quantitative analysis of colcemid-induced chromosomal nondisjunction in Chinese hamster cells in vitro. Cytogenet. Cell Genet. 12: 165-174.

Cox, D.M., and Puck, T.T. 1969. Chromosomal nondisjunction: the action of colcemid on Chinese hamster cells in vitro. Cytogenetics 8: 158-169.

Edwards, R.G. 1954. Colchicine-induced heteroploidy in early mouse embryos. Nature 174: 276-277.

Edwards, R.G. 1958. Colchicine-induced heteroploidy in the mouse. I. The induction of triploidy by treatment of the gametes. J. Exp. Zool. 137: 317-347.

Harrisson, C.M.H., Page, B.M., and Keir, H.M. 1976. Mescaline as a mitotic spindle inhibitor. Nature 260: 138-139.

Jacobs, P.A., and Morton, N.E. 1977. Origin of human trisomics and polyploids. Hum. Hered. 27: $59-72$.

Kamiguchi, Y., Funaki, K., and Mikamo, K. 1976. A new technique for chromosome study of murine oocytes. Proc. Jpn. Acad. 52: 316-319.

Kamiguchi, Y., Funaki, K., and Mikamo, K. 1978. A method for chromosome preparation of murine oocytes and preimplantation embryos. Cong. Anom. (in Japanese). 18: 41-48.

Kamiguchi, Y., Funaki, K., and Mikamo, K. 1979. Chromosomal anomalies caused by preovulatory overripeness of the primary oocyte. Proc. Jpn. Acad. 55: 398-402.

Kato, H., and Yoshida, T.H. 1970. Nondisjunction of chromosomes in a synchronized cell population initiated by reversal of colcemid inhibition. Exp. Cell Res. 60: 459-464.

Kato, H., and Yoshida, T.H. 1971. Isolation of aneusomic clones from Chinese hamster cell line following induction of nondisjunction. Cytogenetics 10: 392-403.

Mattei, J.F., Mattei, M.G., Ayme, S., and Giraud, F. 1979. Origin of the extra chromosome in trisomy 21. Hum. Genet. 46: 107-110.

McGaughey, R.W., and Chang, M.C. 1969. Inhibition of fertilization and production of heteroploidy in eggs of mice treated with colchicine. J. Exp. Zool. 171: 465-480.

Mikamo, K. 1968a. Mechanism of non-disjunction of meiotic chromosomes and of degeneration of maturation spindles in eggs affected by intrafollicular overripeness. Experientia 24: 75-78.

Mikamo, K. 1968b. Intrafollicular overripeness and teratologic development. Cytogenetics 7 : 212-233.

Mikamo, K., and Hamaguchi, H. 1975. Chromosomal disorder caused by preovulatory overripeness of oocytes. In Aging Gametes. R.J. Blandau, ed., S. Karger, Basel, pp. 72-97.

Niikawa, N., Merotto, E., and Kajii, T. 1977. Origin of acrocentric trisomies in spontaneous abortuses. Hum. Genet. 40: 73-78.

Piko, L., and Bomsel-Helmreich, O. 1960. Triploid rat embryos and other chromosomal deviants after colchicine treatment and polyspermy. Nature 186: 737-739.

Wang, R.W., Rebhun, L.I., and Kupchan, S.M. 1977. Antimitotic and antitubulin activity of the tumor inhibitor steganacin. Cancer Res. 37: 3071-3079.

Witschi, E., and Laguens, R. 1963. Chromosomal aberrations in embryos from overripe eggs. Develop. Biol. 7: 605-616. 